\title{
Interdisciplinary Collaborations in the Creation of Digital Dance and Performance: A Critical Examination
}

\author{
Sarah Whatley and Amalia G. Sabiescu
}

\begin{abstract}
This chapter explores the convergence between performance-based cultural heritage and new technologies, with a focus on interdisciplinary collaborations in creation and making processes. These interdisciplinary work spaces present a tremendous potential for innovative art making, as they bring together deep knowledge of the arts and artistic sensibility with a sound understanding of technology languages and possibilities. At the same time, being situated at the confluence of different fields of practice and research dwelling on diverse epistemologies and approaches, interdisciplinary collaborations do more than configure new ways of making art: they contribute to synergies between arts and technology fields, marking places of cross-fertilisation, blurring boundaries and influencing their evolution. Through a close analysis of interdisciplinary undertakings in making digital performance, we show how creative work in mixed teams of performance artists, researchers and practitioners on the one hand, and researchers from technology and design-focused disciplines on the other, is instrumental to the development of what we call interdisciplinary artscapes' and 'interdisciplinary knowledgescapes'. These spaces offer a fertile ground for creative initiatives and knowledge advancement drawing on integrated perspectives, theories, methodologies and approaches from arts and technology fields. Together, interdisciplinary artscapes and interdisciplinary knowledgescapes contribute to opening up and pushing the boundaries of thinking and art making, reconsidering taken for granted assumptions and coming up with radically new art forms.
\end{abstract}

S. Whatley $(\square)$

Coventry University, Priory St, Coventry CV1 5FB, United Kingdom

e-mail: s.whatley@coventry.ac.uk

A.G. Sabiescu

RMIT Europe, C/Minerva 2, Barcelona 08006, Spain

e-mail: amalia.sabiescu@rmit.edu.au 


\section{Introduction}

Performance as cultural practice and performance studies have always been positioned in complex interrelationships with other disciplines. As Dwight Conquergood argues, "(p)erformance studies is uniquely suited for the challenge of braiding together disparate and stratified ways of knowing" (Conquergood 2002: 152). Performance has a multidisciplinary appeal, both as an invitation to study performative acts through the lens of disciplines ranging from history to anthropology, and reversely, lending its own perspectives and paradigms to shed light on processes and phenomena in different fields of study (Madison and Hamera 2005).

This chapter explores the convergence between performance-based cultural heritage and new technologies, with a focus on interdisciplinary collaborations in creation and making processes. Starting from the second half of the twentieth century, when some of the first experiments using computers in performance making were initiated, digital technologies have been employed in different ways to assist, enhance, or completely re-configure the artistic creative process. Artists including choreographers have used digital technologies as choreographic tools, shared working spaces, experimental playgrounds, or have embraced computing languages more broadly to approach their art making, envisaging their artistic work in computational and algorithmic terms. Some of the most innovative creative practices continue to come from interdisciplinary collaborations between performance artists, choreographers, computer scientists, and media artists. These interdisciplinary work spaces present a tremendous potential for innovative art making, as they bring together deep knowledge of the arts and artistic sensibility with a sound understanding of technology languages and possibilities. At the same time, being situated at the confluence of different fields of practice and research dwelling on different epistemologies and approaches, interdisciplinary collaborations do more than configure new ways of making art: they contribute to synergies between arts and technology fields, marking places of cross-fertilisation, blurring boundaries and influencing their mutual evolution.

The chapter offers a critical examination of interdisciplinary collaborations in performance making to shed light on how they are instrumental both for artistic innovation and for fostering knowledge production within and across disciplines. It starts by describing performance and the theorisation of performance as an integrative space, where insights, knowledge, perspectives and approaches from different disciplines can be adopted and employed to enrich understanding of performance acts as well as innovating the art form. This quality of integration is likewise the characteristic feature of interdisciplinarity: 'making whole' by weaving together insights and approaches from different disciplines. We show how interdisciplinary undertakings in performance have a dual edge, blending creative acts and knowledge advancement. Through a close analysis of such undertakings in making digital performance, with a particular focus on dance, we demonstrate how creative work in mixed teams of performance artists, researchers and practitioners on the one hand, and researchers from technology and design-focused disciplines on the other, is instrumental to the development of interdisciplinary artscapes and 
interdisciplinary knowledgescapes: spaces that offer a fertile ground for creative initiatives and knowledge advancement drawing on integrated perspectives, theories, methodologies and approaches from arts and technology fields. Together, interdisciplinary artscapes and interdisciplinary knowledgescapes contribute to opening up and pushing the boundaries of thinking and art making, reconsidering taken for granted assumptions and coming up with radically new art forms.

\section{Performance as an Integrative Space}

Performance is a contested concept, one which has been described from multiple and often conflicting viewpoints (Strine et al. 1990). Historically, it has been categorised variously under the headings of entertainment, show making, a leisure activity, but also as a fundamental cultural activity, one which embodies and expresses worldviews, values and intangible cultural assets that represent group, community and national identities (Madison and Hamera 2005). In this chapter, we look at performance as both a cultural practice and a disciplinary field of research. Performance as 'cultural practice' refers to the cultural rooting of human action or behaviour that is conceived and presented as a performative act. A performance is the expression of ways of knowing, being and cultural identities, and as such it is a window on to and a means of understanding "how human beings fundamentally make culture, affect power, and reinvent their ways of being in the world" (Madison and Hamera 2005: xii). As Schechner (2013) argues, there is basically no limit to what can be considered a performative act, as long as a human activity is "framed, presented, highlighted, or displayed" as such (p. 3). This situates performance across a wide spectrum of human activities and behaviours, ranging from ritual and play to performing arts such as dance and music (Schechner 2013). The focus in this chapter is on performing arts and particularly dance and body-based performance. These forms of performance are also those that most intensely embody and express human culture, as anthropologist Victor Turner notes:

Cultures are most fully expressed in and made conscious of themselves in their ritual and theatrical performances.... A performance is a dialectic of "flow", that is, spontaneous movement in which action and awareness are one, and "reflexivity", in which the central meanings, values and goals of a culture are seen "in action", as they shape and explain behavior. A performance is declarative of our shared humanity, yet it utters the uniqueness of particular cultures. We will know one another better by entering one another's performances and learning their grammars and vocabularies. (Turner 1990: 1)

Performance studies focuses on the study of performance adopting lenses, theories, approaches and methods from a wide range of disciplines, from performing arts to sociology, anthropology, cultural studies and history. At the core of performance studies is the tight relation between practice and research. Many scholars in performance studies are or have been engaged in some kind of performative practice or are experts in specific forms of performance. An actionoriented perspective is also what characterises investigative approaches in performance studies, where: "whatever is being studied is regarded as practices, events, and behaviors, not as 'objects' or 'things"' (Schechner 2013: 3). This confers upon 
performance a "quality of "liveness"' (Schechner 2013) which makes it appealing for scholars in other disciplines who can adopt a performance studies stance or approach to make sense of subjects and objects of research in their own disciplines. To these scholars, performance offers a lens to understand cultural acts, meaning, language, and human behaviour as performances (Madison and Hamera 2005; Schechner 2013).

Performance studies stands out in the academia for its key capacity for integration. This can be seen two ways. Firstly, performance scholars find it easy to borrow and seamlessly employ lenses, perspectives, approaches and theories from other disciplines and integrate them in their object of study. The strong interrelationship with other disciplines is at the heart of performance studies. Performance studies is most active and rich in connections and associations with other disciplines, it is fluid and dynamic, and continues to expand by exploiting interdisciplinary interfaces (Schechner 2013). As Conquergood writes:

The ongoing challenge of performance studies is to refuse and supercede this deeply entrenched division of labor, apartheid of knowledges, that plays out inside the academy as the difference between thinking and doing, interpreting and making, conceptualizing and creating. The division of labor between theory and practice, abstraction and embodiment, is an arbitrary and rigged choice (Conquergood 2002: 153).

Second, performance studies is integrative in its epistemological foundations and premises. Quite uniquely among academic disciplines, performance studies departs from Aristotelian and Cartesian paradigms by its refusal to divorce the mind and the body, the psychological and the somatic in its scientific pursuits. This epistemological stance is particularly vibrant in dance and body-based performance. Dancers' thought processes are intricately bound to a psycho-somatic whole (deLahunta and Zuniga Shaw 2006, 2008). Dancers think through their bodies and can develop and transmit knowledge through gesture and movement. 'Kinaesthetic intelligence', 'physical thinking' are concepts often adopted in dance making practice (deLahunta and Zuniga Shaw 2006). Performance has its own language, which is expressed in movement and thought and words in a space of vibrant liveness and presence:

As performers you are looking for an 'action language': one you can spontaneously 'speak'. ... So you need to think by performing, instead of trying to complete your thinking prior to the performance (Howell 1999: 46).

The flexibility and openness of performance studies makes it uniquely suited for interdisciplinary work. At the same time, its epistemological premises and knowledge-building approaches distinguish it from other disciplines and can raise barriers to productive interdisciplinary dialogue. Performance studies brings to the table a unique way of thinking and meaning making, languages and vocabularies that can be new, obscure or difficult to grasp when seen from the perspective of other disciplines. In the next sections, we examine the premises for interdisciplinary creative practice for digital dance and performance, how it differs from interdisciplinary practice focused uniquely on knowledge building, and raise attention to the importance of duly acknowledging the dynamic interplay between art making and knowledge advancement. 


\section{The Creative Process for Digital Dance and Performance}

The creative process in dance and performance making implies that an idea or a concept is explored creatively. A central creative concept guides choices with respect to movement, performers' exploration of space, the design of costumes, scenic elements, lighting and their evolution in the temporal flow of the performance. Performance creation and production can be described as a 'generative dialogue' between different elements that drive representation and meaning, from movement and lighting to costumes, props and soundscapes (Latulipe et al. 2011). This is a complex and non-linear process in which options and decisions are assessed, taken or refuted until reaching a satisfactory vision. Choreographic thinking underpins rehearsals and devising processes. Ideas are explored and tried out, and changes are brought in a cyclical process to adjust and refine. Handling this complexity requires not only a sense of artistic vision, but also a firm grasp of multiple layers of knowledge covering different aspects of the performance ecology. Even for traditional performances, these knowledges are oftentimes distributed among different individuals who bring their share in the creation and production process. Yet in traditional performances this distributed knowledge ecology is used seamlessly for creative endeavours in a manner which does not reflect the tensions and clashes characteristic of interdisciplinary work. This seamless integration is facilitated by a clear sense of purpose, specific roles and a mutually understood and often taken for granted frame of reference, one which has been established throughout many years of creative practice. For instance, in the Western tradition, the focus of dance performances is on the dancers and their bodily movements as they explore and inhabit the scenic space. Likewise, the creative process is patterned on envisioning and configuring the exploration of space through movement, focusing on the dancers.

With the introduction of digital and interactive technologies, this established process opens up to change. We focus on digital dance and performance in which digital technologies have a pivotal, rather than peripheral role. Examples include virtual reality performances, telematic and distributed performances, online performances, performances which integrate projections, sensing and interactive technologies. Of special interest for our examination are interactive performances, referring broadly to the quality of affording live interaction in the performative space through the mediation of digital technology. The pinnacle of complex interdisciplinary work is interactive performance in which technologies (such as camera tracking and sensor technologies) are used to control or trigger performance components, for instance works where dancers' movements are tracked and generate media projections or sounds in real time (Birringer 2003).

The shifts in the creative process for digital performances are analogous to a changing frame of reference for creative acts. The integration of technology affects the ecosystem in which the performer acts so that spatial connections are reconfigured and, depending on the complexity of the performance, the way bodies and space interact changes fundamentally. Making fairly complex interactive performances requires, therefore, a focus shift from the performer to the 
environment in which the piece is performed, on how the performer relates, reacts to and interacts with technology and the space. We can imagine, for instance, how a traditional dance piece where dancers perform patterned movements exploring the scenic space contrasts with an interactive performance where the movements of the dancers activate sensors which then deliver inputs to trigger soundscapes and digital projections on a screen, in real time. In the first case, the choreographic process focuses on the dancer and sequences of movements and gestures. Lighting, costumes, soundscapes are important elements in the performance ecology, yet decisions regarding their appearance, design and flow throughout the performance are taken to complement the dancers, which are central actors. In an interactive performance with sensing technology, on the other hand, technology becomes one of the principal actors, and the interaction between the dancer and the technology is the main driver of action, audio-visual information and meaning. As Johannes Birringer points out:

Addressing 'interaction' as a spatial and architectural concept for performance, therefore, means shifting the emphasis away from the creation of steps, phrases, 'combinations' or points on the body that initiate movement, away from the dancer's internal bodily awareness (widely encouraged in today's practices of yoga, somatics, experiential anatomy, body-mind centering and release techniques) unto her environment, to a not-given space but a constructed, shifting relational architecture that influences her and that she shapes or that in turn shapes her (Birringer 2003: 90).

This implies embracing a novel paradigm for making dance, away from choreography focused on the movements of the performer towards what Johannes Birringer calls "a relational performance architecture" which moves choreographic thinking into "a plastic process of 'designing' fluid space and responding to transformative space that allows for integration of 'nervous' or sensitive media presences" (2003: 90). The composition process itself is dynamic and evolving, mirroring the emergent nature of the final piece to be developed. Moreover, this process inaugurates a need to access new and complex knowledge about technology, technology design and the interaction paradigms afforded by the technology integration in the scenic space. As performance making becomes entangled with intricate design and engineering processes for designing, testing and integrating seamlessly digital interfaces, interactive systems, and programmed sensors, collaborators develop new vocabularies informed by knowledge of computation capabilities, which can best be advanced by interdisciplinary creative work.

\section{$4 \quad$ Interdisciplinarity in Creative Practice}

The literal meaning of 'interdisciplinary' is 'between fields of study', from the prefix 'inter' meaning 'between, among, in the midst' and 'disciplinary' meaning 'relating to a particular field of study"' (Stember 1991: 4). The increasing academic interest in interdisciplinarity comes from the necessity to investigate questions or issues that cannot be adequately covered by a single disciplinary lens (Repko 2012), or for studying complex systems whose understanding requires bringing together 
diverse analytical perspectives (Newell 2001). An interdisciplinary investigation therefore draws on the outlooks and insights of different disciplines and builds upon them to foster a coherent answer and a comprehensive understanding (Newell 2001; Repko 2012). It is this aspect of integration that distinguishes interdisciplinarity from other investigative approaches that cross the boundaries of a single discipline. Cross-disciplinarity involves the investigation of a phenomenon from the viewpoint and with the tools and approaches of different disciplines, without implying however an integrated approach. One step further, multidisciplinary studies involve scholars from different disciplines working together to achieve a common goal. Their insights and approaches are complementary, without again being necessarily integrated. Interdisciplinarity, on the other hand, refers to a "systematic integration of ideas" (Fiore 2008: 254). Integration, literally "to make whole", implies that "ideas, data and information, methods, tools, concepts, and/or theories from two or more disciplines are synthesized, connected, or blended" (Repko 2012: 4).

This process of integration is captured in the prefix 'inter' and has been interpreted as a three-stage course by Repko (2012):

1. A contested space where issues or problems that cannot be tackled, understood or solved by employing a single disciplinary lens provide the impetus for engaging in interdisciplinary research. The goal is to create something new, whether it is a new theory, a new perspective or a solution to a problem.

2. Acting upon insights, contributions and inputs from various disciplines, in a concurrent, integrative fashion.

3. The result of the integrative process, which can be conceived as an answer, a solution, an intellectual or knowledge advancement.

If interdisciplinary studies focus on the integration of knowledge-related assets and resources, the interdisciplinary work process in the creation of digital dance and performance has a different dynamics, one in which knowledge advancement shadows, supports and uplifts artistic work. We can more closely examine this dynamic by looking further at the three stages outlined above. In the first stage that Repko (2012) identifies, the impetus for collaborative work in interdisciplinary studies can come from the drive to engage with exploring a contested space, find a solution or simply create something new which requires the joint input of people and resources from diverse disciplines. For creative practice, the creation of something new has primacy. Whatever form novelty takes, some instance of knowledge is always involved to make it happen. Some projects may specifically mention knowledge advancement as a specific project goal, along with artistic production. Yet, even when collaborations are uniquely aimed towards art making, knowledge is a pre-requisite, an indispensable ingredient for supporting the foundation of a space of creative possibility. The creative goal and the associated knowledge required further dictates the composition of the teams and the kind of expertise, tools and resources required.

In the second stage, insights from different disciplines are brought together contributing to the creation of the envisaged outcome. In interdisciplinary studies, 
the dynamics of integration plays out around knowledge, tools and resources elicited from the diverse disciplinary traditions involved. In creative practice, the centrality of the creative act pushes knowledge into a subsidiary, yet not least important role. Integration in creative practice therefore refers to blending, braiding or bringing together knowledge, tools, and resources from diverse disciplines to the service of a creative idea. This stage is the crux of the collaborative process and will be examined more closely in the forthcoming section.

In the last stage, outputs are produced. Depending on the goals pursued, these can include finite performances, concepts, ideas, technical tools and systems, choreographic software, but also knowledge, new perspectives and theories. Of particular interest is how these outputs serve the advancement of disciplines or configure new interdisciplinary spaces for knowledge pursuit and art creation processes, which will be discussed in the final section.

\section{The Integrative Process in the Creation of Digital Performance}

This section examines the activities in which interdisciplinary working teams engage, with a focus on 'the integrative process': the moments, approaches and timeframes which delimit the interweaving of interdisciplinary insights and inputs until reaching the desired outcomes. Our goal is to understand what forms, strategies and approaches there are for this process, and further to reflect on how these are instrumental to advancing innovation in art as well as knowledge advancement within and across the disciplines involved. We examine this process by looking at cases from our own research and from the literature, and extracting specific instances to illustrate patterns or strategies for creative work. Some cases are focused on the creation of digital dance and performance, some on the design and development of technology-enhanced tools for creativity, annotation and choreography, while others have a more pronounced knowledge-exchange and sharing component.

The creative process for interactive dance and performance is not unlike non-linear technology design processes, in which conception, design, prototyping and testing are iterated until reaching a satisfactory outcome. The cyclical creation and production pattern is characteristic of highly experimental performances in which very little of the final outcome-concept, choreography, technology, interaction, etc.-is predefined. These types of collaborations have an important exploratory component, and may give equal importance to knowledge advancement as to the actual making of the performance work. Ballectro is an example of a collaborative project into performance and digital media where the goal was to create a staged performance along with researching the interface between performance and new media. Ballectro was a collaboration between the project Assemblages, run by InterMedia at the University of Oslo and the Department of Ballet and Dance at the Oslo National College of the Arts. It aimed to advance understanding not only in the field of performance, but also in the field of technology and design studies, and how 
dance could advance technology design. The creative approach in Ballectro is described as "an experimental, 'free-form' approach to building a collage-like choreographic process" (Skjulstad et al. 2002: 221), expansive, emerging and democratic in nature. Most creative sessions included improvisation tasks in which dancers experimented with digital tools. Apart from the dancers, all the participants in the creative process were invited to improvise, and this included the media and technology researchers. Improvisation was not only a means to a creative output, but also a way to exchange knowledge and learn by reflective practice. The final performance collated fragments from experimental sessions and learning tasks, guided by an evolving choreographic vision during the project course. The research was conducted on a cyclical model, including iterative learning tasks, improvisation sessions, and reflexive activities (Skjulstad et al. 2002).

Improvisational and experimental approaches like Ballectro treat the collaborative space like an experimental playground. The composition process is emergent and dynamic, following the emergent nature of the final piece to be developed. Learning how to work together is a first and vital component. One powerful practice for supporting mutual learning is collaborative rehearsal. The interdisciplinary team assists the enactment of choreographic ideas and concepts, trying out various interaction patterns until configuring desired directions for the composition. Collaborative rehearsals fulfil a variety of learning and creative goals: they enable trying out choreographic ideas, testing technology, and enabling performers to engage with the interactive spaces that are emerging from the composition. As Johannes Birringer comments:

From a choreographic point of view, the dancer within an interactive environment ... will need to familiarize herself with the response behaviour of the sound and video parameters, and both dancer and composer will strive to create an exponentially more sensitive, articulate and intuitive system. In a shared environment this could mean refinements in sensors, filters, and output processors, but also an attenuation of the performer's spatialtemporal consciousness. How is the performer-musician-system relationship evolving, emergent? What can we learn from jazz-improvisational structures, from video game structures, from different cultural contextualizations of virtual environments? (Birringer 2003: 93)

In such improvisational and emergent approaches, roles and spaces of intervention are reconfigured and participants may freely step into the area of expertise of another. As Gonzalez et al. (2012) argue, this is a true instance of an integrated process', when a choreographer may provide vital input for technology design, and in reverse, when technologists may be asked for an opinion regarding the timing of a dance moment. This asks for a continuous process of negotiation, one in which nothing is pre-defined and established hierarchies and role boundaries are blurred. A phenomenon of contagion occurs, new words, phrases, vocabularies and approaches are appropriated and exchanged. This phenomenon enables the configuration of a space of creative possibility from which ideas, concepts and action lines spring forth.

A closer examination of the integrative process in emergent approaches to performance making opens up questions about the interplay between knowledge 
production and creative acts: What kind of knowledge(s) are brought to bear? How do they make their way into creative acts and decisions? How are they shared and what traces to they leave? These aspects are examined by looking at a particularly challenging instance of performance making: working in geographically distant teams to produce a distributed performance.

ULTRAORBISM was a distributed performance designed and developed in the frame of the European project RICHES (Renewal, Innovation and Change: Heritage and European Society), in partnership between the Centre for Dance Research at Coventry University and I2CAT Foundation in Barcelona, with the collaboration of Falmouth University, UK. The aim was to examine, through a real life event, how the integration of digital technology affects performance making, the new expressive means it can afford, and how it changes audience engagement and appreciation of the art form. The performance was a distributed event between Centre d'Art Santa Mònica in Barcelona and Falmouth University, taking place in April 2015.

The concept of the performance was ideated by Marcel-lí Antúnez, a Spanish artist with a rich history of blending performance and interactive technologies. Marcel-lí created a narrative inspired by the travel tale A true story, by Lucian of Samosata (125-180 AD), a travelling rhetorician and satirist who wrote in Ancient Greek. The tale is considered the first account of science fiction, featuring a travel to the moon, but it is also a subtle satire denouncing the mix of fact and fiction in the works of contemporary historians. On this basis, Marcel-lí created a dream-like narrative unfolding through a variety of expressive media, partly developed before the show and partly resulting from the interaction between performers and technology in real time.

The space had a similar configuration in the two locations: an open stage featured the live performers, while animation and video were featured on screens. The performance narrative was projected on the central screen, and alternated between pre-loaded animation and the live performative acts from both locations, with Marcel-lí Antúnez performing in Barcelona, while three dancers and a storyteller performed in Falmouth. Performance details were projected on two smaller screens. The audience in each location could see the happenings in the other location through real-time video playback. Part of the concept of the performance was to make everything visible. Therefore the team of technicians was present, as well as the lighting, sound and remote connection equipment.

ULTRAORBISM is an illustrative case of a distributed, loosely centralised creative process. Whilst the piece was based on a concept by Marcel-lí Antúnez, the performance was fine-tuned and produced jointly by the Catalan-English team of engineers and performers, and tried out during collaborative rehearsals. Setting up collaborative rehearsals between different locations was challenging, especially since rehearsals were not only meant to stage ideas, but to configure and standardize them. The issues raised by making everything work on a technical level for linking and communicating between the two locations were heightened by the fact that there was no outside creative director to take decisions and ensure a smooth flow. While Marcel-lí Antúnez was regarded as the central creative mind behind the 
project, he was also performing, and could not fill the role of a director, able to see the piece unfolding from the outside. A high degree of freedom to propose ideas and make decisions was therefore entrusted to each member of the team. At the same time, the freedom and the lack of hierarchy was demanding, especially for performers, on several levels. Even for decisions that regarded contained actions like the duration of pressing a sensor, performers had to be attentive, aware and knowledgeable of the other elements of the performance and how, together, they created meaning. As one dancer remarked in a post-show focus group, "it is all interconnected": a simple action such as stamping on a sensor affected the ecology of the performance. Moreover, there was also a lack of hierarchy with respect to the various media and expressive components from movement to lighting and projections that together created and communicated meaning. As a dancer pointed out:

What is more important? Is it more important that we are connected so that everyone watching, even if they're separate from us, they feel this united front-right in front of them? Is it more important that we connect to Marcel-1í? Is it more important that we connect to the audience? ... A thousand times we came to a point where we [felt] like we could go down any of these roads and at some point someone has to make a decision (Excerpt from focus group with the ULTRAORBISM Falmouth-based team, 9/04/2015, RICHES project archives).

One of the first aspects of interdisciplinarity to examine in ULTRAORBISM regards the nature and the trajectories of the knowledge elicited throughout the creation and production continuum. Both were configured by the central aim of the project: creating an engaging and immersive distributed performance. Similar to technology design, the artistic creative process can be described as an array of choices dotted on a timeline, which continuously open and close the space of creative or design possibility. In design, these decisions can be called 'framing judgements', choices that continuously open and close, define and redefine "the space of potential design outcomes" (Nelson and Stolterman 2012: 199). These judgements apply to different components of the product or system to be designed, yet eventually they take effect in configuring the product or system as a whole. Analogously, in interactive performances such as ULTRAORBISM, framing judgements are made that regard specific components of the performance, from movement and the timing of movement phrases to technology interaction and lighting; yet these judgements ultimately affect the performance as a whole. Each framing judgement requires a particular knowledge instance, which can be prompted individually or jointly by different members of the team. Knowledge may be verbalised and shared but, especially for performers, it is often tacit, embodied, or so deeply blended with an impulse to act that it is difficult to separate and share. The process of integration at the creative level only requires a portion of this knowledge to be made explicit and shared among the team. For instance, a dancer may sense rather than mentally formulate the exact moment when she should step away from the sensor to keep the harmony in the collective performative act. If the creative goals for the piece are reached through rehearsals, then an explanation of the thinking underpinning the timing and the decision are not 
necessary. Countless decisions such as these are taken during rehearsals-sensed rather than verbalised, and enacted almost at the same time with being thought. If, on the other hand there is a concern with learning and knowledge advancement, then knowledge sharing becomes significant. Instances of tacit knowledge have to be converted in forms that other members of the team can comprehend, while actions and sequences performed spontaneously need to be examined to understand their meaning and significance.

Furthermore, the issue of knowledge traces is significant when considering the legacy of these encounters beyond the lifetime of a project. When used in the service of creative acts, both tacit and explicit knowledge instances have a quality of immediacy, and can be just as ephemeral as the performative act. They are brought into being through experimentation, and may quickly find their way into informing and driving decisions that spur further experimentation until reaching desired forms. Unless purposefully documented, knowledge instances at most echo in the memory of participants, but leave no tangible trace. If the purpose is to encourage joint production and transfer of knowledge among disciplines beyond time-based encounters, then it becomes paramount to document interdisciplinary creative processes. The traces or creative resources resulting from documentation processes are generative, they can be disseminated to inform and inspire future creative and research practice (deLahunta and Zuniga Shaw 2006: 54).

Emergent approaches to making interactive performances can become particularly vital spaces for fostering innovation. Firstly, they foster innovation in the art form, for their capacity to challenge, question and redefine established conventions regarding movement, body, digital media and their interplay. Secondly, they stimulate the production and circulation of knowledge across disciplinary boundaries. By working, experimenting and creating together new perspectives open, and new ways to employ theories, approaches and methodologies come forth. However, to build towards these outcomes, it is necessary to purposefully cultivate knowledge production and sharing along the creative continuum in interdisciplinary practice. In these settings, techniques for knowledge conversion (see for instance Nonaka et al. 2000) and reflection on practice (see Schon 1983) are important for enabling participants to share what they experience and know in tacit ways, and to understand the experience of others. Moreover, documentation of creative practice is important for spreading these knowledges beyond the lifetime of projects and events.

Interdisciplinary collaborations are not restricted to making new performances. A format which recognizes the value of bringing together interdisciplinary experts in performance, dance, media arts and technology design is that of short-term exchange projects, creative and knowledge-exchange workshops and peer to peer labs. These can be called upon to share ideas, reflect upon practice, share works in progress, and devise new concepts and approaches. An early example is the project Software for Dancers (London, 2001), funded by the Arts Council of England and organised with the support of Sadler's Wells and Random Dance Company based in London. The project brought together four choreographers and four digital artists with programming skills to generate ideas and concepts for rehearsal tools that 
could aid in the choreographic practice. The choreographers who took part were Siobhan Davies, Wayne McGregor, Shobana Jeyasingh and Ashley Page. The project used these encounters as an occasion to envisage creative ideas for choreographic tools, but also to examine computational and choreographic approaches to art making, and the importance of understanding the nature of the materials and structures that are integrated and transformed in these processes. The format involved open sessions of discussion, followed by a closer examination of the methods commonly employed by choreographers in their work. Proposals were therefore developed on the concept of a multimedia notebook as a rehearsal tool, and ideas explored the possibility to use the computer as a generative source for choreographic inspiration. Yet the value of the project was less in the outcomes and more in the occasion for interaction and exchange that it provided. The discussions opened up questions about the choreography, the nature of software and code, and how the computer can assist choreographic practice. What are its promises and what its limits?

More recently, the Choreographic Coding Labs (CCLs), initiated in Frankfurt in 2013 and now toured internationally invite creative coders with an interest in movement and choreography to work with dance-related datasets and examine choreographic approaches and structures to advance and innovate their artistic practice. The first CCL was developed through Motion Bank, a 4-year project of the Forsythe Company. The CCLs are invitations to experiment, exchange knowledge and explore new ideas in a stimulating collaborative environment, without aiming for tangible outputs. Despite this open format, outputs are usually produced, ranging from tools for measuring movement qualities to concepts and prototypes for artworks. Some participants come in with works in progress or that they would like to refine, and use the CCL space as an occasion for inspiration and intensive work in a creative atmosphere. A software which grew out of the CCLs and continues to be shaped and refined throughout new editions is PieceMeta, a data management system which enables storing and looping data captured from movement.

The characteristic feature of the CCLs is the peer to peer format, which encourages horizontal learning and exchanges between people who blend technology and arts-related backgrounds and interests. Another aspect is the intensive and concentrated work format. Participants have the chance to explore ideas throughout 5 days against insights and feedback from like-minded peers. Interruptions are occasions for either socialisation or creative input and inspiration. Choreographers and dancers are invited to come and present their work, share their ideas, and be available for questions and discussions. The CCL stands out as a format for dancerelated interdisciplinary exchange and creative practice for its focus on the existing community of creative coders. Participants already possess mixed backgrounds and interests at the junction of arts and computing. Through exposure to dance and choreographic material, new approaches, methods, ideas and ways of thinking cross the arts to the technology domain. As one of the CCL coordinators comments in an interview: 
The CCLs are consistent with my own interest in bringing a high level of dance practice in conjunction with high level digital media arts practice. And my interest is in bringing them together, not necessarily that they make art together, so the choreographers who come and give a talk, they are not there to collaborate with the digital media artists, the goal is not to produce collaborative artwork, necessarily. I mean, collaborations do emerge out of the project, the goal is to try to inform the work of the media artists to give them inspiration coming from dance practice (Interview, 12/01/15, RICHES project archives).

\section{$6 \quad$ Interdisciplinary Artscapes, Interdisciplinary Knowledgescapes}

Intersections and interactions between digital technology and arts fields have now been going on for well over half a century. Impacts on the field of dance and performing arts are notable, yet, some scholars would argue, these are not taking effect at the same rate as for other arts, such as music. As deLahunta (2002) comments, the convergence between performing arts, particularly dance, and technology can be described as episodic or periodic, lacking the breadth and intensity to reverberate in remarkable, foundation-shattering impacts. In their being episodic and by engaging a finite number of actors, their impacts are reduced in scale. Yet, we argue, there is more to these interdisciplinary encounters than their tangible, project-bound outcomes. To understand how their impact builds up in time it is useful to look at the process of integration, characteristic of interdisciplinary work, not only at micro, but also at macro-scale. At micro-scale, interdisciplinary research is mostly driven forward by teams of researchers belonging to different disciplines working on common subjects, projects or issues. At macro-scale, when consistent and enduring interdisciplinary work gains critical mass, it can lead to the emergence of new, interdisciplinary constructs, theories, approaches and techniques and eventually lay the foundation of new interdisciplines, solidified by the foundation of new professional roles, academic departments and curricula. This process of integration going from the micro to macro-scale has been described by Klein (1996) with reference to three landmark steps: (1) Detaching a research subject from its disciplinary frameworks; (2) completing the gaps left opened by single discipline investigation; and (3) redefining boundaries and founding new "knowledge spaces and new professional roles" (Klein 1996: 36-37). These are processes happening over a long period of time, and demonstrate the high level of fluidity and dynamism of knowledge advancement through interdisciplinary research. Disciplines are not fixed, they grow and change and influence one another and often redefine their boundaries and hierarchies, such that a new interdiscipline can become in time a well established discipline in its own right (Repko 2012).

The process of integration happens simultaneously at micro and macro-scales, influencing and feeding into each other. The more different types of interdisciplinary encounters concentrate on a timeline, the greater impetus and momentum is created for new, interdisciplinary spaces that blend the thinking, resources, theories, and methodologies of diverse fields. The interfaces between arts and technology 
fields explored as part of these encounters gradually come to be concretised in spaces rich with potential for creativity, artistic innovation and knowledge advancement. Given the tight interplay between theory and practice, research and arts making, macro-scale developments for arts and technology collaborations can be conceived as the gradual configuration of intertwined and mutually influencing interdisciplinary artscapes and interdisciplinary knowledgescapes. The first concept captures the emergence of spaces of creative possibility that draw insights, resources, tools and inspiration from manifold domains, from performance to design, human-computer interaction and software engineering. The latter are spaces that blend different epistemological and disciplinary approaches, insights and theories in ways that cannot be afforded within specific disciplinary confines.

At present, interdisciplinary artscapes and knowledgescapes for performance and technology intersections exist more as potential than as reality. To come into effect, there is a need to reinforce both their immaterial dimension (made of knowledge, approaches, theories and ways of thinking) and their material dimension (made of physical or represented counterparts of the former, as well as research and practice infrastructures and new generations of practitioners and researchers with an interdisciplinary training). At the moment, most contributions coming from interdisciplinary collaborations are in the field of dance and performance rather than digital media studies, design, and human-computer interaction. One of the most notable impacts involves the adoption of perspectives, frameworks and concepts borrowed from technology disciplines. Technological developments can inform conceptions of the body, movement, and gestuality. In a "technological epistemology of the body", the metaphor of the machine or computer is used to illustrate how the body functions (deLahunta 2004: 236). Further, new ways of thinking about movement, choreography and composition in media terms emerge. For instance, as early as 1975, the dance pieces Locus and Accumulation by choreographer Trisha Brown provide instructions for movement which can be seen as a source code, one which can be replicated. The instructions for Accumulation read:

The accumulation is an additive procedure where movement 1 is presented; start over. Movement 1; 2 is added and start over. 1, 2, 3 is added and start over, etc., until the dance ends (cited in deLahunta 2003: 306).

Second, the performing arts domain benefits from the creation of software tools that can aid choreographers in their creative process. Such tools were typically created by artists in arts organisations who had programing skills and an early concern with using technology to innovate creative processes (deLahunta 2005). Some of these tools had a short lifespan and were used only experimentally, others provided inspiration for artists to continue to experiment and innovate, while others, such as Life Forms (made by a USA-based research team with the contribution of the dancer and choreographer Merce Cunningham), and Isadora (a software tool that assists the creation of interactive performances, made by artistprogrammer Mark Coniglio) were adopted by artists and continue to be used to this day. These tools are not neutral, they can influence the work and affect the way 
the creator is thinking about their own making practice. They are therefore instrumental to adopting and appropriating ways of thinking, meaning making, and composition algorithms that are characteristic of the technology field.

Moreover, collaborations between performance artists and technologists contribute to radical innovation in the art form. The last two decades in particular saw the emergence of new forms of performance, whether theatre (head-phone theatre, installation theatre, digital theatre, Internet theatre) or dance and body-based performance (Wearables for performance, telematics, networked performance, screendance). There are other, more subtle influences migrating from the technology to the arts field, having to do with the endorsement of attitudes, approaches and visions for making art, even philosophical or axiological principles. In his essay Open source choreography? deLahunta (2003) comments on the parallels between the Open Source movement and the increasing interest among dance practitioners and choreographers to make available documentation that illustrates their practice and creative work. This interest is driven to some extent by principles that echo those animating the Open Source movement and having to do with an ethos of free sharing and reuse. Yet unlike open software, which is free to use and modify and is effectively a property of the commons, the collective pool of information on dance making, while freely available, is still attached to frameworks and regulations that privilege individual, rather than collective, authorship.

On the other hand, the contribution of performance to technology fields is still underexplored. The potential is there to inform both new ways of thinking about technology, as well as informing methodologies for digital media design and interpretation (Skjulstad et al. 2002). The premises and promises that performing arts paradigms and ways of thinking could bring to computer technologies were sketched more than two decades ago, and found a vibrant expression in Brenda Laurel's book Computers as theatre (2013). The book examines how computer activities can be seen from a perspective grounded in theatre and television studies, and envisages how human-computer interaction can cater for more engaging user experiences by looking into approaches to playwriting and audience engagement. The book opened a new page in the interplay between theatre and computing, one which is still being written. As Don Norman points out in the Foreword to the 2013 edition:

Theatre is about interaction, about themes and conflicts, goals and approaches to those goals, frustration, success, tension, and then the resolution of that tension. Theatre is dynamic, changing, always in motion. Our modern technologies with their powerful computers, multiple sensors, communication links, and displays are also about interaction, and treating that interaction as theatre proves to be rich, enlightening and powerful. (Norman 2013: xi).

Still unfolding is also the configuration of the new interdisciplinary spaces of knowledge and art development, which interdisciplinary collaborations in performance making are contributing to. The potential, in these new spaces, is to give rise to new literacies, new ways of imagining interactions between body, movement and computing technologies, and sketching new premises for the creation of innovative 
art. While there has been a significant amount of research on new literacies, digital and multimodal, little research exists on the role of dance and performance in informing these new literacies (Skjulstad et al. 2002).

\section{Conclusion}

This chapter provided a critical examination of interdisciplinary collaborations in making digital performances, seeking to articulate their contribution to advancing both art making and knowledge production within and across disciplines. Such interdisciplinary creative practice is very varied and can be oriented towards making new performances, designing and developing technical systems and tools, coming up with new concepts, ideas, and theories, or sharing and developing knowledge across disciplines. Whilst these encounters are mostly episodic, often organised in the frame of time-bound projects, their impact on disciplinary growth and arts innovation is cumulative. The field of performance, by its nature open to integration and novel perspectives, gains new understandings and approaches to art making through the appropriation of technical or design-informed approaches, methodologies and conceptual lenses. In reverse, technical and design disciplines can be informed by performance studies in their interpretation of technology and human-machine interactions, and in devising new theoretical and methodological pathways for innovative interaction and software design. Moreover, interdisciplinary collaborations contribute to configuring what we have called interdisciplinary artscapes and interdisciplinary knowledgescapes: spaces in between which offer new premises, resources, tools, theories and methodologies for making and theorising art drawing on integrative perspectives bridging arts and technology fields. Analogous to the tight interplay between theory and practice in performance studies, interdisciplinary artscapes (as integrative spaces of creative possibility) and knowledgescapes (as integrative knowledge and meaning-making spaces) are tightly intertwined, mutually influencing each others' evolution. Because of this quality of integration, their greatest potential is to develop and offer new languages, vocabularies, paradigms, and literacies, and in time configure radically new ways of making and theorising arts and culture.

Open Access This chapter is distributed under the terms of the Creative Commons AttributionNoncommercial 2.5 License (http://creativecommons.org/licenses/by-nc/2.5/) which permits any noncommercial use, distribution, and reproduction in any medium, provided the original author(s) and source are credited.

The images or other third party material in this chapter are included in the work's Creative Commons license, unless indicated otherwise in the credit line; if such material is not included in the work's Creative Commons license and the respective action is not permitted by statutory regulation, users will need to obtain permission from the license holder to duplicate, adapt or reproduce the material. 


\section{References}

Birringer, J. (2003). Dance and interactivity. Dance Research Journal, 35(2), 89-111.

Conquergood, D. (2002). Performance studies: Interventions and radical research. The Drama Review, 46(2), 145-156.

deLahunta, S. (2002). Periodic convergences: Dance and computers. In Tanz und technologiel dance and technology (pp. 66-84). Berlin, Germany: Alexander Verlag.

deLahunta, S. (2003). Open source choreography? In G. Stocker \& C. Schöpf (Eds.), Code: The language of our time (pp. 304-310). Ostfildern, Germany: Hatje Cantz Publishers.

DeLahunta, S. (2004). L'Appareil de Locomotion: Une Épistémé Technologique. Interagir avec les technologies numériques: Nouvelles de Danse, 52, 36-49.

deLahunta, S. (2005). Isadora: An interview with artist/programmer Mark Coniglio. International Journal of Performance and Digital Media, 1(1), 31-46.

deLahunta, S., \& Zuniga Shaw, N. (2006). Constructing memories: Creation of the choreographic resource. Performance Research, 11(4), 53-62.

deLahunta, S., \& Zuniga Shaw, N. (2008). Choreographic resources agents, archives, scores and installations. Performance Research, 13(1), 131-133.

Fiore, S. M. (2008). Interdisciplinarity as teamwork: How the science of teams can inform team science. Small Group Research, 39(3), 251-277.

Gonzalez, B., Carroll, E., \& Latulipe, C. (2012). Dance-inspired technology, technology-inspired dance. In Proceedings of the 7th Nordic Conference on Human-Computer Interaction: Making Sense Through Design (pp. 398-407). New York: ACM.

Howell, A. (1999). The analysis of performance art. Amsterdam: Harwood Academic Publishers.

Klein, J. T. (1996). Crossing boundaries: Knowledge, disciplinarities, and interdisciplinarities. Charlottesville: University of Virginia Press.

Latulipe, C., Wilson, D., Huskey, S., Gonzalez, B., \& Word, M. (2011). Temporal integration of interactive technology in dance: creative process impacts. In Proceedings of the 8th ACM Conference on Creativity and Cognition (pp. 107-116). New York: ACM.

Laurel, B. (2013). Computers as theatre. Upper Saddle River, NJ: Addison-Wesley.

Madison, D. S., \& Hamera, J. (2005). Introduction: Performance studies at the intersections. In D. S. Madison \& J. A. Hamera (Eds.), The Sage handbook of performance studies (pp. xi-xxv). Thousand Oaks, CA: Sage Publications.

Nelson, H. G., \& Stolterman, E. (2012). The design way: Intentional change in an unpredictable world: Foundations and fundamentals of design competence. Englewood Cliffs, NJ: Educational Technology.

Newell, W. H. (2001). A theory of interdisciplinary studies. Issues in Integrative Studies, 19(1), $1-25$.

Nonaka, I., Toyama, R., \& Konno, N. (2000). SECI, Ba and leadership: A unified model of dynamic knowledge creation. Long Range Planning, 33(1), 5-34.

Norman, D. (2013). Foreword to Laurel, B. Computers as theatre: A dramatic theory of interactive experience (2nd ed., pp. xi-xv). Upper Saddle River, NJ: Addison-Wesley.

Repko, A. F. (2012). Interdisciplinary research. Process and theory. Thousand Oaks, CA: Sage Publications.

Schechner, R. (2013). What is performance studies? Rupkatha Journal on Interdisciplinary Studies in Humanities, 5(2), 2-11. Special Issue on Performance Studies.

Schon, D. A. (1983). The reflective practitioner. New York: Basic books.

Skjulstad, S., Morrison, A., \& Aaberge, A. (2002). Researching performance, performing research: Dance, multimedia and learning. In A. Morrison (Ed.), Researching ICTs in context (pp. 211-248). Oslo, Norway: InterMedia, University of Oslo.

Stember, M. (1991). Advancing the social sciences through the interdisciplinary enterprise. Social Science Journal, 28(1), 1-14. 
Strine, M. S., Long, B. W., \& Hopkins, M. F. (1990). Research in interpretation and performance studies: Trends, issues, priorities. In G. M. Phillips \& J. T. Wood (Eds.), Speech communication: Essays to commemorate the 75th anniversary of the Speech Communication Association (pp. 181-204). Carbondale: Southern Illinois University Press.

Turner, R. (1990). Introduction. In W. Appel \& R. Schechner (Eds.), By means of performance. Intercultural studies of theatre and ritual (pp. 1-7). Cambridge, MA: Cambridge University Press. 